Tôhoku Math. Journ.

20 (1968), 199-206.

\title{
A STRUCTURE THEOREM OF AUTOMORPHISMS OF VON NEUMANN ALGEBRAS
}

\author{
TAKATERU OKAYASU
}

(Received November 30, 1967)

In a recent few years automorphisms and derivations of $C^{*}$-algebras, especially of von Neumann algebras, have been investigated by various authors [1], [6], [7], [8], [11] and etc. We also are concerned with them in this paper. Our main purpose is to establish a structure theorem of (not necessarily *-preserving) automorphisms of von Neumann algebras, which we may call the polar decomposition theorem. It asserts that any automorphism of a von Neumann algebra is composed of an inner automorphism defined by an invertible positive operator and a *automorphism in the unique way. This fact seems to assure us that the study of automorphisms may be reduced, in a sense, to that of *-preserving ones. For example, it can be said that the property that an automorphism is outer is due to its *-preserving part. Also we know, as its immediate consequence, that any automorphism of a von Neumann algebra is $\sigma$-strongly (that is, in the strongest operator-topology), as well as $\sigma$-weakly, bicontinuous. These arguments can be applied to those of isomorphisms between von Neumann algebras. Particularly we can give an answer to a problem proposed by $\mathrm{S}$. Sakai in his lecture note [10], deciding that any isomorphism between von Neumann algebras is $\sigma$-strongly bicontinuous.

The author wishes to express here his gratitude to Dr. M. Takesaki for his helpful suggestions.

1. Preliminaries. It is assumed that all $C^{*}$-algebras under consideration have identities. Automorphisms, isomorphisms and representations of $C^{*}$-algebras are said to be *-automorphisms, *-isomorphisms and *-representations, respectively, if they are *-preserving.

Let $H$ be a Hilbert space and $B(H)$ the $C^{*}$-algebra of all bounded linear operators on $H$. If an operator $a \in B(H)$ is invertible, $\rho_{a}(x)=a x a^{-1}$ for $x \in B(H)$ is clearly an automorphism of $B(H)$. When $A$ is a $C^{*}$-algebra acting on $H$, an automorphism $\rho$ of $A$ is said to be spatial if there is an invertible operator $a \in B(H)$ such that $\rho=\rho_{a} \mid A$, the restriction of $\rho_{a}$ on $A$. A spatial automorphism $\rho$ of $A$ is said to be inner if the above $a$ can be chosen in $A$ and to be outer if it is not inner. An automorphism $\rho$ of $A$ is said to be weakly inner ( $\pi$-inner in [8]) if for any faithful *-representation $\pi$ of $A$ there 
is an inner automorphism $\sigma$ of the weak closure $\widetilde{\pi(A)}$ of $\pi(A)$ in $B(K)$ such that $\pi \circ \rho \circ \pi^{-1}=\sigma \mid \pi(A)$, where $K$ denotes the representation space of $\pi$.

The following theorem asserts that all spatial *-automorphisms are defined by unitaries :

THEOREM 1. Let $A$ be a $C^{*}$-algebra on a Hilbert space $H$, a an invertible bounded linear operator on $H$ such as $\rho_{a}(A) \cong A$. If $\rho_{a} \mid A$ is a *automorphism of $A$, then there is a unitary $u \in B(H)$ such as $\rho_{a}\left|A=\rho_{u}\right| A$.

Moreover, if $A$ is a von Neumann algebra and if a belongs to $A$, then $u$ can be chosen in $A$.

PROOF. By the hypotheses,

$$
a x a^{-1}=\rho_{a}(x)=\left[\rho_{a}\left(x^{*}\right)\right]^{*}=\left(a^{*}\right)^{-1} x a^{*} ;
$$

and hence,

$$
a^{*} a x=x a^{*} a,
$$

for all $x \in A$. Then $a^{*} a$ is contained in the commutator $A^{\prime}$ of $A$. Let $a=u h$ be the polar decomposition of $a$, where $u$ is a unitary and $h=\left(a^{*} a\right)^{1 / 2}$ an invertible operator which is in $A^{\prime}$. Therefore

$$
\rho_{u}(x)=a h^{-1} x h a^{-1}=a x a^{-1}=\rho_{a}(x)
$$

for all $x \in A$.

The second assertion is seen by remarking that, under the additional conditions, $u$ is contained in $A$ as well as $h$ is in the center of $A$. q.e.d.

LEMMA 2. Let $H$ be a Hilbert space, $a \in B(H)$ an invertible operator. Then, $\mathrm{S}_{\mathrm{p}}(\cdot)$ denoting the spectrum,

$$
\mathrm{S}_{\mathrm{p}}\left(\rho_{a}\right) \subseteq \mathrm{S}_{\mathrm{p}}(a) \mathrm{S}_{\mathrm{p}}(a)^{-1}=\left\{\lambda \mu^{-1} ; \lambda, \mu \in \mathrm{S}_{\mathrm{p}}(a)\right\}
$$

PROOF. For each $b \in B(H)$ let us concider the operators $l_{b} x=b x$ and $r_{b} x=x b$ for $x \in B(H)$. The mapping $b \rightarrow l_{b}$ is an isomorphism of $B(H)$ into the Banach algebra of all bounded linear operators on $B(H)$ and the mapping $b \rightarrow r_{b}$ is an anti-isomorphism. Then $\mathrm{S}_{\mathrm{p}}\left(l_{b}\right) \subseteq \mathrm{S}_{\mathrm{p}}(b)$ and $\mathrm{S}_{\mathrm{p}}\left(r_{b}\right) \subseteq \mathrm{S}_{\mathrm{p}}(b)$. Since $\left(r_{a}\right)^{-1}=r_{a-1}$ and $l_{a}\left(r_{a}\right)^{-1}=\left(r_{a}\right)^{-1} l_{a}=\rho_{a}$, we have

$$
\mathrm{S}_{\mathrm{p}}\left(\rho_{a}\right)=\mathrm{S}_{\mathrm{p}}\left(l_{a}\left(r_{a}\right)^{-1}\right) \cong \mathrm{S}_{\mathrm{p}}\left(l_{a}\right) \mathrm{S}_{\mathrm{p}}\left(r_{a}\right)^{-1} \cong \mathrm{S}_{\mathrm{p}}(a) \mathrm{S}_{\mathrm{p}}(a)^{-1}
$$

If a linear operator $\delta$ on a $C^{*}$-algebra $A$ satisfies $\delta(x y)=\delta(x) y+x \delta(y)$ for each $x, y \in A$, it is called a derivation of $A$. That derivations are necessarily continuous is known. It is clear that, for $a \in B(H)$, ad $a(x)=a x-x a, x \in B(H)$ 
is a derivation of $B(H)$. When a $C^{*}$-algebra $A$ acts on $H$, a derivation $\delta$ of $A$ is said to be spatial if there is an operator $a \in B(H)$ such as $\delta=\operatorname{ad} a \mid A$. The meanings of inner derivations, outer derivations and weakly inner derivations should be taken similarly as for those of automorphisms. A result of $\mathrm{S}$. Sakai [11] (see [6], [8] also) asserting that each derivation of a von Neumann algebra is necessarily inner is remarkable and will play an important role in the following discussions. Using the method of the proof of Lemma 2, we can show

LEMMA 3. Let $H$ be a Hilbert space and a a bounded linear operator on H. Then,

$$
\mathrm{S}_{\mathrm{p}}(\mathrm{ad} a) \subseteq \mathrm{S}_{\mathrm{p}}(a)-\mathrm{S}_{\mathrm{p}}(a)=\left\{\lambda-\mu ; \lambda, \mu \in \mathrm{S}_{\mathrm{p}}(a)\right\} .
$$

A derivation $\delta$ on a $C^{*}$-algebra $A$ is said to be skew-adjoint if it satisfies $\delta\left(x^{*}\right)=-\delta(x)^{*}$ for all $x \in A$.

LEMMA 4. Let a be a bounded linear operator on a Hilbert space H. If $a$ is self-adjoint, then ada is skew-adjoint; and conversely if ada is skewadjoint, then there is a self-adjoint operator $b \in B(H)$ such as $\operatorname{ad} b=\operatorname{ad} a$. Moreover if $a$ is in a $C^{*}$-algebra $A$ on $H$, then $b$ mentioned above can be chosen in $A$.

ProOF. The first half is easily verified; its converse is seen by, for a given $a$, putting $b=\left[a^{*}+a\right] / 2$, together with the last assertion. q.e.d.

2. Automorphisms. We denote by Log the principal analytic continuation of the log onto the domain $C \backslash\{-\infty<t \leqq 0\}$ obtained by excluding the negative half-axis $\{-\infty<t \leqq 0\}$ from the complex plane $C$.

THEOREM 5 (cf.[4]). Let $H$ be a Hilbert space, $S$ a closed subspace of $B(H)$. If $a \in B(H)$ has its spectrum in $\{\lambda ; \operatorname{Re}(\lambda)>0\}$ and satisfies $\rho_{a}(S) \subseteq S$, then $\operatorname{ad}(\log a)(S) \subseteq S$ and $\rho_{\exp (t \mathrm{Log} a)}(S)=S$ for any real $t$.

PROOF. Let us put $b=\log a$ and $\sigma_{t}=\rho_{\exp (t))}$ for each real $t$, then the mapping $t \rightarrow \sigma_{t}$ becomes a norm-continuous one-parameter group of operators of $B(H)$ into itself. Since $[\exp (t b)-1] / t$ converges to $b$ uniformly as $t$ converges to 0 , we have, $\iota$ denoting the identity automorphism of $B(H)$,

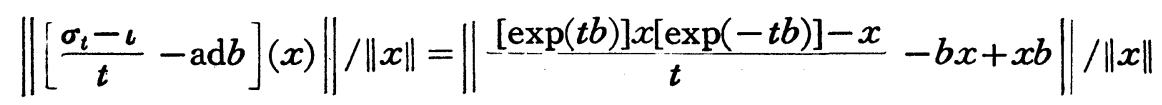

$$
\begin{aligned}
& =\|[\exp (t b)-1] x \frac{1-\exp (t b)}{t} \exp (-t b)+x\left[\frac{1-\exp (t b)}{t}+b\right] \exp (-t b)
\end{aligned}
$$




$$
\begin{array}{r}
-x b[\exp (-t b)-1]+\left[\frac{\exp (t b)-1}{t}-b\right] x\|/\| x \| \\
\leqq\|\exp (t b)-1\|\left\|\frac{1-\exp (t b)}{t}\right\|\|\exp (-t b)\|+\left\|\frac{1-\exp (t b)}{t}+b\right\|\|\exp (-t b)\| \\
+\|b\|\|\exp (-t b)-1\|+\left\|\frac{\exp (t b)-1}{t}-b\right\| \rightarrow 0,
\end{array}
$$

as $t \rightarrow 0$. Therefore we have $\sigma_{t}=\exp (\operatorname{tad} b)$, in particular $\rho_{a}=\exp (\operatorname{ad} b)$ (see[3], p.283, Theorem 9.4.2). Since $\mathrm{S}_{\mathrm{p}}\left(\rho_{a}\right) \subseteq \mathrm{S}_{\mathrm{p}}(a) \mathrm{S}_{\mathrm{p}}(a)^{-1} \leqq C \backslash\{-\infty<t \leqq 0\}$ and $\mathrm{S}_{\mathrm{p}}(\mathrm{ad} b)$ $\cong \mathrm{S}_{\mathrm{p}}(b)-\mathrm{S}_{\mathrm{p}}(b) \leqq\{\lambda ;|\operatorname{Im}(\lambda)|<\pi\}$, we know $\log \rho_{a}=$ ad $b$ by Lorch's theorem which determines the period of $\exp ([9])$. The theorem of Runge enables us to choose a sequence of polynomials which converges to Log uniformly in the wider sense, hence $\log \rho_{a}$ is a uniform limit of polynomials of $\rho_{a}$. Thus, by the assumption, $\operatorname{ad} b(S) \subseteq S$ and also $\rho_{\exp (t b)}(S) \subseteq S$ for any $t$. The latter gives $\rho_{\exp (t b)}{ }^{-1}(S)=\rho_{\exp (-t b)}(S) \subseteq S$, so that $\rho_{\exp (t b)}(S)=S$.

q.e.d.

THEOREM 6. Let $H, K$ be Hilbert spaces, $M$ a von Neumann algebra on $H, A$ a $C^{*}$-algebra on $K$ and $\pi$ an isomorphism of $M$ onto $A$. If $a \in B(K)$ with $\mathrm{S}_{\mathrm{p}}(a) \subseteq\{\lambda ; \operatorname{Re}(\lambda)>0\}$ satisfies $\rho_{a}(A) \subseteq A$, then there is an invertible operator $b$ in $M$ such as $\pi^{-1} \circ \rho_{a} \circ \pi=\rho_{b} \mid M$.

In particular, if $\pi$ is $a *$-isomorphism of $M$ onto $A$ and if $a \in B(K)$ is an invertible positive operator, we can choose $b$ to be invertible and positive.

PROOF. By Theorem $5, t \rightarrow \tau_{t}=\pi^{-1} \circ \rho_{\exp (t \log a)} \circ \pi$ becomes a norm-continuous one-parameter group of automorphisms of $M$. Moreover, by the similar culculation to that in the proof of Theorem 5 ,

$$
\left\|\frac{\tau_{t}-\iota}{t}(x)-\pi^{-1}[a \mathrm{~d}(\log a)(\pi(x))]\right\| /\|x\| \longrightarrow 0
$$

as $t \longrightarrow 0$. Since the mapping $M \ni x \longrightarrow \pi^{-1}[\operatorname{ad}(\log a)(\pi(x))]$ becomes a derivation of $M$, by S. Sakai's result, there is an operator $c$ in $M$ such as $\pi^{-1}[\operatorname{ad}(\log a)(\pi(x))]=\operatorname{ad} c(x)$ for all $x \in M$. Let us put $\tau_{t}^{\prime}=\rho_{\text {exp }(t \mathrm{adc})} \mid M$ for each real $t$. Then, by $\left\|\frac{\tau_{t}^{\prime}-\iota}{t}-\operatorname{ad} c \mid M\right\| \longrightarrow 0$ as $t \longrightarrow 0$, we have $\tau_{t}^{\prime}=\exp$ $(\operatorname{tad} c) \mid M=\tau_{t}$, especially

$$
\rho_{b} \mid M=\pi^{-1} \circ \rho_{a} \circ \pi,
$$

where $b=\exp c$, which is of course in $M$.

If $\pi$ is a $*$-isomorphism and $a$ an invertible positive operator, $M \ni x \longrightarrow$ $\pi^{-1}[\operatorname{ad}(\log a)(\pi(x))]$ becomes a skew-adjoint derivation of $M$, then again by 
Sakai's result and by Lemma 4, we can choose a self-adjoint operator $c \in M$ such as $\pi^{-1}[\operatorname{ad}(\log a)(\pi(x))]=\operatorname{ad} c(x)$ for all $x \in M$. Putting $b=\exp c \in M$ as above, we have $\rho_{b} \mid M=\pi^{-1} \circ \rho_{a} \circ \pi$.

q.e.d.

As a direct corollary, we have a sufficient condition for a spatial automorphism of a von Neumann algebra to be inner.

COROllaRy 7. Let $H$ be a Hilbert space, $M$ a von Neumann algebra on $H$. If $a \in B(H)$ has its spectrum in $\{\lambda ; \operatorname{Re}(\lambda)>0\}$ and satisfies $\rho_{a}(M) \cong M$, then the spatial automorphism $\rho_{a} \mid M$ defined by $a$ is inner.

Now we state the polar decomposition theorem for automorphisms of von Neumann algebras. It will be extended to $C^{*}$-algebras in a natural sense using the concept "weakly inner automorphisms".

THEOREM 8. Let $H$ be a Hilbert space, $M$ a von Neumann algebra on $H, \rho$ an automorphism of $M$. Then there are inner automorphisms $\rho_{1}, \rho_{1^{\prime}}$ defined by invertible positive operators in $M$ and *automorphisms $\rho_{2}, \rho_{2}^{\prime}$ such as $\rho=\rho_{2} \circ \rho_{1}=\rho_{1}{ }^{\prime} \circ \rho_{2}{ }^{\prime}$. Suppose that $\bar{\rho}_{1}, \bar{\rho}_{1}{ }^{\prime}$ are inner automorphisms defined by invertible positive operators in $M$ and $\bar{\rho}_{2}, \bar{\rho}_{2}^{\prime}{ }^{*}$-automorphisms such as $\rho=\bar{\rho}_{2} \circ \bar{\rho}_{1}=\bar{\rho}_{1}^{\prime} \circ \bar{\rho}_{2}^{\prime}$, then $\rho_{1}=\bar{\rho}_{1}, \rho_{2}=\overline{\rho_{2}}$ and $\rho_{1}^{\prime}=\overline{\rho_{1}}, \rho_{2}^{\prime}=\overline{\rho_{2}}$.

PROOF. Let $\alpha$ be the greatest atomic representation of $M$, that is, the direct sum of all irreducible *-representations of $M, L$ its representation space. It is well-known that $\alpha$ is a ${ }^{*}$-isomorphism. Hence $\sigma=\alpha \circ \rho \circ \alpha^{-1}$ becomes an automorphism of $A=\alpha(M)$. Because for each maximal left ideal $I$ in $M$ the quotient Banach space $M / I$ becomes a Hilbert space ([12]; also [5]) and $\rho$ carries $I$ to a maximal left ideal $\rho(I)$, we can define an invertible linear operator $s_{I}$ of $M / I$ to $M / \rho(I)$ by

$$
s_{1}(x+I)=\rho(x)+\rho(I) .
$$

Here $\left\|s_{I}\right\| \leqq\|\rho\|$ and $\left\|\left(s_{I}\right)^{-1}\right\| \leqq\left\|\rho^{-1}\right\|$ hold. We can consider that $L$ is a direct sum of $\{M / I\}_{\mathrm{I}}$ with suitable multiplicities and define an invertible bounded linear operator $s$ as the direct sum of $s_{I}^{\prime}$ 's with corresponding multiplicities. Then the formula $\sigma=\rho_{s} \mid A$ can be observed. Let $s=v h$ be the polar decomposition, where $v$ is a unitary and $h=\left(s^{*} s\right)^{1 / 2}$ an invertible positive operator. Then for any $b \in A$,

$$
h^{-2} b h^{2}=\left(s^{*} s\right)^{-1} b\left(s^{*} s\right)=s^{-1}\left(s^{-1}\right)^{*} b s^{*} s=s^{-1}\left(s b^{*} s^{-1}\right)^{*} s=\sigma^{-1}\left(\sigma\left(b^{*}\right)^{*}\right)
$$

is contained in $A$, therefore $\rho_{h^{-s}}(A) \subseteq A$; and hence by Theorem 5 , 


$$
\rho_{h^{-1}}(A) \subseteq A \text { and } \rho_{h}(A) \cong A
$$

also for any $b \in A$,

$$
v b v^{-1}=s h^{-1} b h s^{-1}=\sigma^{\prime}\left(\rho_{h^{-1}}(b)\right) \in A,
$$

in other words, $\rho_{v}(A) \subseteq A$ (cf. Lemma 4.6 in [4]). We define here

$$
o_{1}=\rho_{h}\left|A, o_{2}^{\circ}=\rho_{v}\right| A ; \rho_{1}=\alpha^{-1} \circ \sigma_{1}^{+} \circ \alpha, \rho_{2}=\alpha^{-1} \circ \sigma_{2} \circ \alpha .
$$

Then, by Theorem $6, \rho_{1}$ is an inner automorphism which is defined by an invertible positive operator in $M$; and trivially $\rho_{2}$ is a ${ }^{*}$-automorphism. Because of $\sigma=\sigma_{2}^{*} \circ \sigma_{1}$, we have

$$
\rho=\alpha^{-1} \circ \sigma^{\circ} \circ \alpha=\left(\alpha^{-1} \circ \sigma_{2} \circ \alpha\right) \circ\left(\alpha^{-1} \circ o_{1}^{\circ} \circ \alpha\right)=\rho_{2} \circ \rho_{1} .
$$

An analogous argument using the polar decomposition $s^{*}=w^{*} k$ of $s^{*}$, where $k=\left(s s^{*}\right)^{1 / 2}$ and $w$ a unitary, leads us to $\rho=\rho_{1}{ }^{\prime} \circ \rho_{2}{ }^{\prime}$.

Next we show the uniqueness of the decomposition. For our purpose it is sufficient to see that if $h_{1}$ and $h_{2}$ are invertible positive operators in $M$ and if $\rho_{h_{2}} \circ \rho_{h_{1}}\left|M=\rho_{r_{2} i_{1}}\right| M$ is *-preserving then $\rho_{r_{2}} \circ \rho_{h_{1}} \mid M$ is just the identity automorphism $\iota \mid M$ of $M$. Since

$$
h_{2} h_{1} x h_{1}^{-1} h_{2}^{-1}=\left[\rho_{h_{2} h_{1}}\left(x^{*}\right)\right]^{*}=h_{2}^{-1} h_{1}{ }^{-1} x h_{1} h_{2}
$$

for all $x \in M, h_{1} h_{2}{ }^{2} h_{1}$ is contained in the center $Z$ of $M$. Let $h_{2} h_{1}=u h$ be the polar decomposition of $h_{2} h_{1}$, where $u$ is a unitary and $h$ an invertible positive operator. Then, first

$$
h^{2}=h_{1} h_{2}{ }^{2} h_{1} \in Z, \text { hence } h \in Z ;
$$

and secondly

$$
h_{1} h_{2} h^{-1}=u^{*}=u^{-1}=h_{1}^{-1} h_{2}^{-1} h
$$

then $h_{1}{ }^{2}=h_{2}{ }^{-2} h^{2}$, hence $h_{1}=h_{2}{ }^{-1} h$.

Therefore $h_{2} h_{1}=h \in Z$, and

$$
\rho_{h_{\iota}} \circ \rho_{h_{1}}\left|M=\rho_{h}\right| M=\iota \mid M .
$$

q.e.d.

Since any spatial automorphism and any *automorphism of a von 


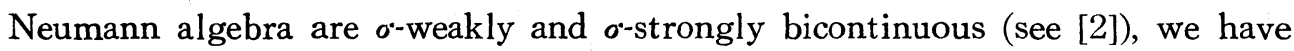

THEOREM 9. If $\rho$ is an automorphism of a von Neumann algebra $M$ on a Hilbert space $H$, then $\rho$ is $\sigma$-weakly and $\sigma$-strongly bicontinuous.

3. Isomorphisms. The following theorem will be shown by the same method employed in Theorem 8:

THEOREM I. Let $H, K$ be Hilbert spaces, $M, N$ von Neumann algebras on $H, K$, respectively, $\rho$ an isomorphism of $M$ onto $N$. Then there are inner automorphisms $\rho_{1}$ of $M, \rho_{1}^{\prime}$ of $N$ which are defined by invertible

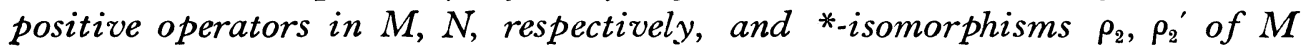
onto $N$ such as $\rho=\rho_{2} \circ \rho_{1}=\rho_{1}^{\prime} \circ \rho_{2}^{\prime}$. Furthermore this decomposition is unique.

It has been known that if an isomorphism $\rho$ of a von Neumann algebra

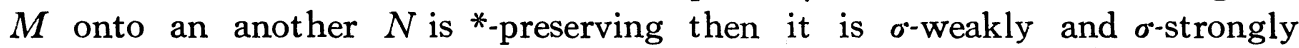
bicontinuous (J. Dixmier [2]); and that an isomorphism of $M$ onto $N$ is $\sigma$-weakly bicontinuous and on a bounded part $\sigma^{*}$-strongly bicontinuous (S.Sakai [11]). Then it is natural to raise a question, as on p.1.52 in [11], whether an isomorphism is $o$-strongly bicontinuous. The answer is immediate from Theorem I :

THEOREM II. Let $H, K$ be Hilbert spaces, $M, N$ von Neumann algebras on $H, K$, respectively, $\rho$ an isomorphism of $M$ onto $N$. Then $\rho$ is $\sigma$-weakly and o-strongly bicontinuous.

4. Addendum. When this paper had been almost prepered, the author had a chance to see a preprint of S.Sakai's article [14] in which it had been shown that any derivation on a simple $C^{*}$-algebra with an identity is inner. Using this and following along the same line of the arguments in section 2 , we can state

THEOREM III. Let $A$ be a simple $C^{*}$-algebra with an identity, $B$ a $C^{*}$-algebra, $\rho$ an isomorphism of $A$ onto $B$. Then there are inner automorphisms $\rho_{1}$ of $A, \rho_{1}^{\prime}$ of $B$ which are defined by invertible positive operators in $A, B$, respectively, and *-isomorphisms $\rho_{2}, \rho_{2}^{\prime}$ of $A$ onto $B$ such as $\rho=\rho_{2} \circ \rho_{1}=\rho_{1}{ }^{\circ} \circ \rho_{2}^{\prime}$. Furthermore this decomposition is unique.

In [13] the auther found a similar result to our Theorem 5 .

\section{REFERENCES}

[1] H. Borchers, Energy and momentum observables in quantum field theory, Comm. Math. Phys., 2 (1966), 49-54. 
[ 2 ] J. DixMIER, Les algèbres d'opérateurs dans l'espace hilbertien, Gauthier villars, 1957.

[ 3 ] E. HILle AND R. S. PhIllips, Functional analysis and semi-groups, Amer. Math. Soc., Providence, 1957.

[ 4 ] L. GARDNER, On isomorphisms of $C^{*}$-algebras, Amer. Journ. Math., 87 (1965), 384396.

[ 5 ] R. KAdison, Irreducible operator algebras, Proc. Nat. Acad. Sci. U.S. A., 43(1957), $273-276$

[6] R. KADISON, Derivations of operator algebras, Ann. Math, 83(1966), 280-293.

[ 7 ] R. KADISON AND J. RINGROSE, Derivations of operator group algebras, Amer. Journ. Math., 88 (1966), 562-576.

[8] R. KADISON and J.RINGROSE, Derivations and automorphisms of operator algebras, Comm. Math. Phys., 4(1967), 32-63.

[ 9 ] E. R. LORCH, The theory of analytic functions in normed abelian vector rings, Trans. Amer. Math. Soc., 54(1943), 414-425.

[10] S. SAKAI, The theory of $W^{*}$-algebras, Lecture note at Yale Univ., New Haven, 1962.

[11] S. SAKAI, Derivations of $W^{*}$-algebras, Ann. Math., 83(1966), 273-279.

[12] M. TAKESAKI, On the conjugate space of operator algebra, Tôhoku Math. Journ., 10 (1958), 194-203.

[13] G. Zeller-Meier, Sur les automorphisms des algèbres de Banach, C. R. Acad. Sci. Paris, 264 (1967), 1131-1132.

[14] S.SAKAI, Derivations of simple $C^{*}$-algebras, to appear.

COllege of General Education

TÔHOKU UNIVERSITY

SENDAI, JAPAN 